\title{
Verfassungsfragen der Personalvertretung im Deutschen Bundestag ${ }^{*}$
}

\author{
Bodo Pieroth und Andreas Meßmann
}

Im Rahmen einer gerichtlichen Streitigkeit um die Gültigkeit der Wahl eines Personalrats der Beschäftigten der SPD-Bundestagsfraktion hat das Verwaltungsgericht Berlin entschieden, dass „die Anwendung des Bundespersonalvertretungsgesetzes wohl näher liegen (dürfte) als die des Betriebsverfassungsgesetzes" 1 . Es hat ausdrücklich einer Entscheidung des Arbeitsgerichts Berlin² widersprochen, wonach auf Fraktionen des Bundestages das Betriebsverfassungsgesetz Anwendung finden solle.

Die gleiche Frage stellt sich bei den Mitarbeitern ${ }^{3}$, die gemäß $₫ 12$ Abs. 3 Abgeordnetengesetz (AbgG) von den Bundestagsabgeordneten beschäftigt werden. Deren Mitbestimmung wird gegenwärtig in einer nach Fraktionen unterschiedlichen Weise praktiziert: Teils gibt es eine personalvertretungs-, teils eine betriebsverfassungsrechtliche, teils gar keine Mitbestimmung. Zutreffend ist in Parallele zu der Entscheidung des Verwaltungsgerichts Berlin die Anwendbarkeit des Bundespersonalvertretungsgesetzes (BPersVG) festgestellt worden, was an anderer Stelle näher begründet worden ist. ${ }^{4}$ Dann stellt sich aber die weitere Frage, ob Verfassungsnormen der Anwendung des BPersVG auf die Mitarbeiter von Bundestagsabgeordneten entgegenstehen. Diese Frage soll im Folgenden untersucht werden.

\section{Behinderungsverbot gemäß Art. 48 Abs. $2 G G$}

\subsection{Abstrakter Inhalt}

Die Rechtsprechung des Bundesverfassungsgerichts hat Art. 48 Abs. 2 GG so verstanden, dass darunter nur solche Verhaltensweisen fallen, die die Übernahme oder Ausübung des Mandats erschweren oder unmöglich machen sollen, nicht aber in andere Richtung zielende Handlungen, die unvermeidlicherweise eine Beeinträchtigung der Mandatsfreiheit als tatsächliche Folge oder Wirkung nach sich ziehen. ${ }^{5}$ Diese so genannte Absichtsformel wird

* Der Beitrag ist aus einem Teil des Rechtsgutachtens hervorgegangen, das der Erstverfasser im Oktober 2008 der Kommission des Ältestenrats des Deutschen Bundestages für Angelegenheiten der Mitarbeiter erstattet hat. Die Verfasser danken dem Justitiariat des Bundestages für die Zustimmung zu dieser Veröffentlichung.

1 Verwaltungsgericht Berlin, Beschluss vom 30. September 2008, VG 72 A 5.08, S. 6.

2 Vgl. Arbeitsgericht Berlin, Urteil vom 17. Januar 2003, 96 Ca 30440/02, in: NZA-RR 2003, S. 656; zustimmend Reinhard Singer, Der mitbestimmungsrechtliche Status der Parlamentsfraktionen, in: NZA 2008, S. 789 - 795, S. 793 ff.

3 Der Ausdruck wird zur Vereinfachung gewählt; er umfasst selbstverständlich auch Mitarbeiterinnen, ebenso wie der Bundestagsabgeordnete auch für die Bundestagsabgeordnete steht.

4 Vgl. Bodo Pieroth / Andreas Meßmann, Betriebsverfassungs- oder personalvertretungsrechtliche Mitbestimmung der Mitarbeiter von Bundestagsabgeordneten?, in: Die Personalvertretung, 52. Jg. (2009), H. 12, S. $444-453$.

5 Vgl. BVerfGE 42, S. 312, S. 329; dem folgend BVerwGE 73, S. 263, S. 282; BVerwGE 76, S. 157, S. 170; BVerwGE 86, S. 99, S. 118; BGHZ 94, S. 248, S. 251. 
in der Literatur zu Recht überwiegend abgelehnt. ${ }^{6}$ Sie ergibt sich weder aus dem Wortlaut des Art. 48 Abs. 2 GG noch aus dessen Sinn und Zweck; denn der Schutz des Abgeordneten vor anderen soll objektiv gewährleistet sein und nicht von mentalen Eigenschaften desjenigen abhängen, gegen den der grundrechtliche Schutz gerichtet ist. Der Wortlaut des Satzes 2 in Art. 48 Abs. 2 GG gibt den entscheidenden Hinweis für die richtige Interpretation des Satzes 1; Satz 2 wird allgemein als ein Unterfall von Satz 1 angesehen. Wenn danach eine Kündigung oder Entlassung „aus diesem Grunde“ unzulässig sein soll, dann wird auf die Ausübung des Amtes eines Abgeordneten verwiesen. Für „aus diesem Grunde“ kann man auch synonym „wegen“ setzen. Richtig verstanden verbietet also Art. 48 Abs. 2 GG Behinderungen wegen der Ausübung des Mandats.

Für seine Auslegung gilt danach dasselbe, was das BVerfG zu dem Merkmal „wegen“ in Art. 3 Abs. 3 S. 1 GG entschieden hat. Auch zu dieser Norm hatte das Gericht zunächst eine Absichtsformel verwendet: Die Diskriminierungsverbote sollen „nicht absolut“ gelten und „nur die bezweckte Benachteiligung oder Bevorzugung“ verbieten, „nicht aber einen

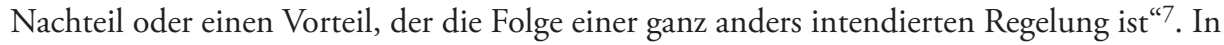
seiner neueren Rechtsprechung hat sich das BVerfG aber von der Absichtsformel gelöst und die Diskriminierungsverbote so ausgelegt, dass die verpönten Merkmale nicht als rechtfertigende Gründe für Ungleichbehandlungen taugen. ${ }^{8}$ Dementsprechend sind Behinderungen im Sinne des Art. 48 Abs. 2 GG alle diejenigen, die gerade wegen der Ausübung des Mandats erfolgen, oder genauer: Eine Handlung ist dann keine Behinderung im Sinne des Art. 48 Abs. 2 GG, wenn ihre Geeignetheit und Notwendigkeit begründet werden kann, ohne dass auf die Ausübung des Mandats abgestellt wird. ${ }^{9}$ Diese normative Aussage wird hauptsächlich gegenüber Privaten relevant, die dem Abgeordneten gegenüber nachteilige Handlungen vornehmen. Insoweit kommt Art. 48 Abs. 2 GG wie auch Art. 38 Abs. 1 S. 2 GG Drittwirkung zu. ${ }^{10}$

\subsection{Konkrete Anwendung}

Das BPersVG bezweckt nicht, die Übernahme oder Ausübung eines Bundestagsmandats zu erschweren oder unmöglich zu machen. Auch können die Geeignetheit und Notwendigkeit betrieblicher Mitbestimmung gerechtfertigt werden, ohne dass auf die Ausübung des Mandats abgestellt wird. Deshalb steht der Mitbestimmung weder nach der Rechtsprechung des Bundesverfassungsgerichts noch nach der vorzugswürdigen Auslegung des Schrifttums das Behinderungsverbot (Art. 48 Abs. 2 GG) entgegen.

6 Vgl. nur Hans Herbert von Arnim, in: Rudolf Dolzer / Wolfgang Kahl / Christian Waldhoff / Karin Graßhof (Hrsg.), Bonner Kommentar zum Grundgesetz, Loseblattsammlung, Heidelberg 2010, Art. 48 Rn. 38; Hans H. Klein, in: Theodor Maunz / Günter Dürig (Hrsg.), Grundgesetz. Kommentar, Loseblattsammlung, München 2010, Art. 48 Rn. 85; Siegfried Magiera, in: Michael Sachs (Hrsg.), Grundgesetz. Kommentar, München 2010, Art. 48 Rn. 11.

7 BVerfGE 75, S. 30, S. 70; dem folgend BVerwGE 75, S. 86, S. 96.

8 Vgl. BVerfGE 85, S. 191, S. 206 f.; BVerfGE 89, S. 276, S. 288 f.

9 Vgl. Bodo Pieroth / Bernhard Schlink, Grundrechte. Staatsrecht II, Heidelberg 2010, Rn. 481.

10 Vgl. Hans H. Klein, a.a.O. (Fn. 6), Art. 48 Rn. 109. 


\subsection{Abstrakter Inhalt}

Art. 38 Abs. 1 S. 2 GG, der die Rechtsstellung der Bundestagsabgeordneten regelt, hat einen Doppelcharakter: Er ist zum einen eine Aufgabenzuweisung an die Träger eines „Amts“ (vgl. den Wortlaut des Art. 48 Abs. 2 S. 1 GG). Als Teil des Bundestags üben die Abgeordneten öffentliche Gewalt aus. Damit korrespondiert, dass das BVerfG schon früh entschieden hat, dass Art. 38 Abs. 1 S. 2 GG anders als sein Satz 1 kein grundrechtsgleiches Recht darstellt. ${ }^{11}$ Für die Wahrnehmung des Amts ist besonders relevant, dass die Abgeordneten Vertreter des ganzen Volkes sind (Art. 38 Abs. 1 S. 2 GG). Die Aufgabenwahrnehmung des Abgeordneten ist also weder auf die Interessen seines Wahlkreises noch gar auf die einzelner Personen oder Personengruppen innerhalb seines Wahlkreises beschränkt. Das heißt aber nicht, dass der Abgeordnete sich nicht der Interessen seines Wahlkreises und auch einzelner Personen oder Personengruppen besonders annehmen darf, wenn er dies als wichtig für das Gesamtvolk ansieht. ${ }^{12}$ Zum anderen hat Art. 38 Abs. 1 S. 2 GG den Charakter eines subjektiven öffentlichen Rechts ${ }^{13}$, das die Abgeordneten schützt. Der Grundstatus der Freiheit des Abgeordneten bedeutet einen Schutz vor allen staatlichen Maßnahmen, die den Bestand und die Dauer des Mandats beeinträchtigen und die inhaltliche Bindung der Mandatsausübung herbeiführen oder sanktionieren. Verfassungsgemäße Gesetze gehören nicht zu solchen Maßnahmen, weshalb die alleinige Gewissensunterworfenheit nicht von der Beachtung der Rechtsordnung entbindet. ${ }^{14}$ Darüber hinaus bedeutet der Grundstatus einen entsprechenden Schutz auch gegenüber Privaten, insbesondere Parteien und Wählern. ${ }^{15}$ In der Verwendung der Begriffe „Aufträge“ und „Weisungen“ kann man diese doppelte Schutzrichtung ausgedrückt sehen, da „Aufträge“ vornehmlich zivilrechtlich und „Weisungen“ vornehmlich öffentlich-rechtlich verwendet werden; aber weithin hält man diese Begriffe für eine (verstärkende) Tautologie. ${ }^{16}$

Im vorliegenden Zusammenhang geht es vor allem um die Rechtswirkungen dieses Schutzes. Schon aus Art. 38 Abs. 1 S. 2 GG folgt, dass rechtliche Bindungen bezüglich der Ausübung des Mandats zwischen dem Abgeordneten und Privaten nicht zustande kommen können, dass es aber unabhängig davon faktische Bindungen gibt. ${ }^{17}$ Das führt bei Rechtshandlungen, deren Rechtsfolge gerade die Bindung ist, wie Verträge und verpflichtende

11 Vgl. BVerfGE 6, S. 445, S. 448.

12 Vgl. Theodor Maunz, in: ders. / Günter Dürig, a.a.O. (Fn. 6), Art. 38 Rn. 11; Norbert Achterberg / Martin Schulte, in: Hermann von Mangoldt / Friedrich Klein / Christian Starck (Hrsg.), Das Bonner Grundgesetz, München 2005, Art. 38 Rn. 39.

13 Vgl. Bodo Pieroth, in: Hans Dieter Jarass / ders., Grundgesetz. Kommentar, München 2009, Art. 38 Rn. 25, 36.

14 Vgl. Hans-Peter Schneider, in: Erhard Denninger / Wolfgang Hoffmann-Riem / Hans-Peter Schneider / Ekkehard Stein (Hrsg.), Kommentar zum Grundgesetz, Loseblattsammlung, Neuwied 2002, Art. 38 Rn. 40.

15 Vgl. Bodo Pieroth, a.a.O. (Fn. 13), Art. 38 Rn. 27; Hans-Peter Schneider, a.a.O. (Fn. 14), Art. 38 Rn. 39.

16 Vgl. Martin Morlok, in: Horst Dreier (Hrsg.), Grundgesetz. Kommentar, Bd. II, Tübingen 2006, Art. 38 Rn. 146; Hans-Heinrich Trute, in: Ingo von Münch / Philip Kunig (Hrsg.), GrundgesetzKommentar, Bd. II, München 2001, Art. 38 Rn. 86.

17 Vgl. Christian Wefelmeier, Repräsentation und Abgeordnetenmandat, Stuttgart 1991, S. 164. 
Erklärungen, zu deren Nichtigkeit; bei Rechtshandlungen, die auch ohne Bindung rechtlich sinnvoll bleiben, wie Beschlüsse von Parteitagen, führt die Freiheit des Abgeordneten dazu, dass sie ihnen gegenüber keine Bindungswirkung entfalten. ${ }^{18}$ Von Verträgen und verpflichtenden Erklärungen sind einseitige Erklärungen des Abgeordneten über sein parlamentarisches Verhalten, insbesondere Wahlversprechungen oder Wahlzusagen („pledges") und politische Absichtserklärungen zu unterscheiden. Diese sind nicht nur faktisch möglich, sondern auch rechtlich zulässig, allerdings nach dem Gesagten rechtlich wirkungslos beziehungsweise unverbindlich. ${ }^{19}$ Die Freiheit des Abgeordneten kann auch ein sachlicher Grund für die Befristung eines Arbeitsverhältnisses zu einem wissenschaftlichen Mitarbeiter sein, zu dessen Aufgaben gerade die fachliche Beratung und politische Bewertung zählen. ${ }^{20}$

Unmittelbar aus der verfassungsrechtlichen Verbürgung der Freiheit des Abgeordneten (Art. 38 Abs. 1 S. 2 GG) folgt daher anerkanntermaßen die Unzulässigkeit folgender Handlungen: Zum einen dürfen keine rechtlichen Bindungen über den Bestand und die Dauer des Mandats herbeigeführt werden, wie bedingte Annahme der Wahl, Blankoverzichtserklärungen ${ }^{21}$ und Rücktrittsreverse. Zum anderen sind rechtliche Verpflichtungen über die grundsätzliche Ausübung des Mandats unzulässig, insbesondere Stimmenkaufverträge und Verpflichtungen finanzieller Art (zum Beispiel Rückzahlungsversprechen, Schuldscheine) für den Fall des Parteienwechsels ohne Mandatsniederlegung. ${ }^{22}$

\subsection{Konkrete Anwendung}

Durch die Mitbestimmung nach dem BPersVG wird jedenfalls nicht unmittelbar in die legislative Tätigkeit des Abgeordneten eingegriffen, denn die Abstimmungen im Bundestag oder seine sonstige parlamentarische Arbeit unterliegen nicht der Mitbestimmung nach dem BPersVG.

Allerdings können Fragen der Einstellung ( $\$ 75$ Abs. 1 Nr. 1 BPersVG) und Kündigung $(\$ 79$ BPersVG) von Abgeordnetenmitarbeitern über deren Arbeitszeit ( $\$ 75$ Abs. 3 Nr. 1 BPersVG) mitbestimmungs- beziehungsweise mitwirkungspflichtig sein oder auch die Anhörung des Personalrats erfordern. Dadurch kommt diesem als Organ der Mitbestimmung zwar kein unmittelbarer Einfluss auf die parlamentarische Arbeit des Bundestagsabgeordneten zu. Gerade wegen der nur geringen Anzahl seiner Mitarbeiter ist der Abgeordnete für eine effektive Ausübung seiner Tätigkeit aber auf jeden von ihnen angewiesen. Die Verzögerung der Kündigung eines unwilligen, unfähigen oder mit dem Abgeordneten (politisch) zerstrittenen Mitarbeiters kann den Abgeordneten daher durchaus in der Ausübung seines Mandats faktisch beeinträchtigen. Außerdem kann bei plötzlich eintretenden neuen politischen Sachlagen oder der unvorhergesehenen Übernahme neuer parlamentarischer Funktionen etwa in einem Fachausschuss oder ähnlichen auch die kurzfristige Einstellung eines Referenten mit einschlägigen Kenntnissen für eine effektive Ausübung des Mandats not-

18 Vgl. Bodo Pieroth, a.a.O. (Fn. 13), Art. 38 Rn. 27.

19 Vgl. Hans-Peter Schneider, a.a.O. (Fn. 14), Art. 38 Rn. 25, 39.

20 Vgl. BAG, in: NZA 1999, S. 149, S. 150 f.

21 Vgl. BVerfGE 2, S. 1, S. 74.

22 Vgl. Hans-Peter Schneider, a.a.O. (Fn. 14), Art. 38 Rn. 39; Hans-Heinrich Trute, a.a.O. (Fn. 16), Art. 38 Rn. 87, 102. 
wendig sein. Schließlich ist der Abgeordnete in politischen Krisen möglicherweise jenseits der regelmäßigen Arbeitszeiten auf seine Mitarbeiter angewiesen.

Durch die Mitbestimmung beziehungsweise Mitwirkung in diesen Fragen und durch die damit zuweilen einhergehenden Verzögerungen kommt dem Personalrat daher ein wenigstens mittelbar-faktischer Einfluss auf die Ausübung des Mandats des Bundestagsabgeordneten zu. Fraglich ist, ob das bereits die Annahme einer Kollisionslage rechtfertigt. Das wäre nur dann der Fall, wenn diese Beeinträchtigung eine solche Intensität und ein solches Gewicht aufweist, dass sie nicht nur als bloße Belästigung oder Bagatelle, sondern als Eingriff in das freie Mandat zu qualifizieren ist. ${ }^{23}$ Diese Grenze ist gerade bei Art. 38 Abs. $1 \mathrm{~S}$. 2 GG schwer zu ziehen ${ }^{24}$, und ihre Überschreitung kommt hier unter mehreren Gesichtspunkten in Betracht.

\section{(a) Effektivität der Mandatsausübung}

Eine Kollisionslage lässt sich nicht bereits deshalb bejahen, weil eine allfällige mittelbarfaktische Beeinträchtigung des Abgeordneten möglicherweise die Effektivität seiner Mandatsausübung wie oben beschrieben negativ beeinflusst. ${ }^{25}$ Art. 38 Abs. 1 S. 2 GG garantiert nämlich nur eine von sachlichen und persönlichen Abhängigkeiten freie Mandatsausübung ${ }^{26}$, dispensiert aber nicht von der Bindung an die allgemein geltenden Gesetze. ${ }^{27}$ Die mit deren Beachtung notwendig einhergehende Verlangsamung der parlamentarischen Arbeit nimmt das Grundgesetz deshalb grundsätzlich genauso hin wie den Zeitverlust, den die Grundentscheidung für das Freie im Gegensatz zum imperativen Mandat bei der parlamentarischen Arbeit bedeutet. Eine Beeinflussung der Effektivität des Abgeordneten durch die Befolgung der allgemein geltenden Gesetze verletzt deshalb nicht etwa das Freie Mandat aus Art. 38 Abs. 1 S. 2 GG, sondern ist letztlich Ausdruck der Bindung aller staatlicher Gewalt an Recht und Gesetz. Wollte man schon jede Beeinträchtigung der Effektivität der Amtsführung durch allgemein geltende Gesetze als Verletzung von Art. 38 Abs. 1 S. 2 GG ansehen, wäre das Freie Mandat beispielsweise auch durch die Straßenverkehrsordnung ( $\mathrm{StVO})$ beeinträchtigt, wenn ein Abgeordneter nur noch unter Überschreitung der dort festgesetzten Höchstgeschwindigkeiten rechtzeitig zu einer Plenarsitzung gelangen, also nur unter Verletzung der StVO sein Amt effektiv ausüben könnte.

23 Zur vergleichbaren Problematik bei der Abgrenzung von Belästigungen und Bagatellen zu mittelbar-faktischen Grundrechtseingriffen vgl. Bodo Pieroth / Bernhard Schlink, a.a.O. (Fn. 9), Rn. $257 \mathrm{ff}$.

24 Vgl. Norbert Achterberg / Martin Schulte, a.a.O. (Fn. 12), Art. 38 Rn. 40 mit weiteren Nachweisen.

25 Die allgemeine Frage, ob das Personalvertretungsrecht die Funktionsfähigkeit der Verwaltung in verfassungsrechtlich relevanter Weise beeinträchtigt, verneint das BVerfGE 93, S. 37, S. 84 mit Blick auf straffe Fristen, Abkürzungsmöglichkeiten und die Möglichkeit vorläufiger Regelungen. Bejahend hingegen Werner Rob, Mitbestimmung im Staatsdienst im Lichte der Strukturprinzipien des demokratischen, sozialen Rechtsstaats, Bad Neuenahr 1998, S. $344 \mathrm{ff}$.

26 Vgl. Norbert Achterberg / Martin Schulte, a.a.O. (Fn. 12), Art. 38 Rn. 40; Bodo Pieroth, a.a.O. (Fn. 13), Art. 38 Rn. 26.

27 Vgl. Theodor Maunz, a.a.O. (Fn. 12), Art. 38 Rn. 17; Martin Morlok, a.a.O. (Fn. 16) Art. 38 Rn. 147 mit weiteren Nachweisen. 
(b) Entscheidungsfreiheit des Abgeordneten

Eine Kollision der Mitbestimmung mit dem Freien Mandat kann allenfalls dann vorliegen, wenn durch die Mitbestimmung von Art. 38 Abs. 1 S. 2 GG gerade die verfassungsrechtlich geschützten Rechtspositionen des Abgeordneten beeinträchtigt werden. Zu diesen gehört in erster Linie die Entscheidungsfreiheit bei der parlamentarischen Arbeit, das heißt die sachliche und persönliche Freiheit des Abgeordneten bei der Ausübung seiner parlamentarischen Rechte. ${ }^{28}$ Die Mitbestimmungstatbestände des BPersVG verpflichten ihn aber nicht zu einer bestimmten Ausübung seiner parlamentarischen Informations- und Teilhaberechte. Sie wirken allenfalls dadurch mittelbar-faktisch auf die vor der Rechtsausübung liegende Willensbildung des Abgeordneten ein, dass sie etwa die Einstellung oder Trennung von Mitarbeitern beziehungsweise die Anordnung von Überstunden verzögern. Die (Entscheidungs-)Freiheit des Abgeordneten selbst bleibt von der Mitbestimmung unberührt.

Dem könnte man entgegenhalten, dass das Bundesarbeitsgericht (BAG) gerade wegen des Einflusses bestimmter Mitarbeiter auf die Willensbildung des Abgeordneten dessen Freiheit und die aus ihr folgende Freiheit der Fraktionen als sachlichen Grund für die Befristung eines Arbeitsvertrages eines wissenschaftlichen Mitarbeiters ansieht:

„Fraktionen werden von den Abgeordneten des für die jeweilige Legislaturperiode gewählten Parlaments gebildet. Das führt nach jeder Wahl zu Änderungen in der personellen Zusammensetzung einer Fraktion. Fraktionen sind, ebenso wie die in ihnen zusammengeschlossenen Abgeordneten, frei in ihrer Entscheidung, Inhalt und Ziel ihrer politischen Arbeit zu bestimmen. Dazu müssen sie nach ihrer Neukonstituierung jeweils entscheiden können, von welchen wissenschaftlichen Mitarbeitern sie sich künftig beraten und ihre parlamentarische Arbeit unterstützen lassen wollen. Diesem verfassungsrechtlich verbürgten parlamentarischen Teilhaberecht trägt die Befristung des Arbeitsverhältnisses eines wissenschaftlichen Mitarbeiters Rechnung. Die dadurch gesicherte Unabhängigkeit der Mandatsausübung schließt eine Umgehung kündigungsrechtlicher Bestimmungen durch den Zeitvertrag aus. Dieser besondere Sachgrund rechtfertigt allerdings nur die Befristung derjenigen wissenschaftlichen Mitarbeiter, deren Aufgabe darin besteht, die Fraktion durch fachliche Beratung und politische Bewertung zu unterstützen. Bei anderen Fraktionsmitarbeitern, etwa im Büro- oder Verwaltungsbereich, vermag dieser Sachgrund die Befristung nicht zu tragen.

Nach den Feststellungen des LAG erfüllt der Kläger als wissenschaftlicher Mitarbeiter für Wirtschaftspolitik diese Voraussetzungen. [...] Zwar bestimmte die Fraktion beziehungsweise deren Wirtschaftspolitischer Sprecher die von dem Kläger zu bearbeitenden Themenstellungen. Dennoch wirkte er durch seine Beiträge und seine Aufbereitung der Themenbereiche inhaltlich an der politischen Willensbildung der Fraktion mit. Dafür ist nicht das Ausmaß der individuellen Einflussnahme eines wissenschaftlichen Fraktionsmitarbeiters entscheidend, sondern dass er aufgrund seiner Funktion die politische Willensbildung der Fraktion beeinflussen kann." 29

Hieraus ergibt sich jedoch nur ein sachlicher Grund für eine Befristung und - wenn man die Rechtsprechung des BAG weiterdenkt - möglicherweise auch ein sachlicher Grund für die Kündigung eines wissenschaftlichen Mitarbeiters. Das BAG erkennt durch seine Rechtsprechung also nur an, dass das Freie Mandat durch die unbefristete beziehungsweise fort-

28 Vgl. oben 2.1.

29 BAG, in: NZA 1999, S. 149, S. 150 f. 
gesetzte (Weiter-)Beschäftigung eines wissenschaftlichen Mitarbeiters trotz einer politischen Neuausrichtung des Abgeordneten wegen seines besonderen Einflusses auf dessen Willensbildung ${ }^{30}$ beeinträchtigt würde.

Des Weiteren sieht das BAG das Freie Mandat durch die unbefristete beziehungsweise fortgesetzte (Weiter-)Beschäftigung von Mitarbeitern ohne oder mit nur geringem Einfluss auf die Willensbildung des Abgeordneten, etwa Sekretariats- und Verwaltungsmitarbeiter, nicht gefährdet. Unter diesem Aspekt besteht daher bei solchen Mitarbeitern erst Recht kein Grund, eine Beeinträchtigung des Freien Mandats durch die bloß verzögernden Regelungen des BPersVG zu befürchten. Zudem kommt den mit der Mitbestimmung, Mitwirkung beziehungsweise Anhörung des Personalrats verbundenen Verzögerungen schon aufgrund ihrer vergleichsweise kurzen Dauer auch bei „politischen“ Abgeordnetenmitarbeitern kein solches Gewicht im Hinblick auf die Willensbildung des Abgeordneten zu wie der unbefristeten oder fortgesetzten (Weiter-)Beschäftigung. ${ }^{31}$ Und schließlich kann der Abgeordnete einem seine Freiheit beeinträchtigenden Einfluss eines Mitarbeiters auf seine Willensbildung während des Mitbestimmungs-, Mitwirkungs- oder Anhörungsverfahrens durch vorläufige Regelungen nach $\$ \$ 69$ Abs. 5, 72 Abs. 6 BPersVG begegnen. Denn wenn das Freie Mandat ein sachlicher Grund für die Befristung eines Arbeitsverhältnisses ist, dann duldet eine Maßnahme, die eine Beeinträchtigung der Willensbildung des Abgeordneten und damit eine Verletzung der Freiheit des Mandats verhindern soll, der Natur der Sache nach keinen Aufschub und ermächtigt den Abgeordneten deshalb zu vorläufigen Regelungen.

Die Entscheidungsfreiheit des Abgeordneten wird also auch nicht insoweit beeinträchtigt, als es durch mögliche Verzögerungen durch die Mitbestimmung im öffentlichen Dienst zu einem mittelbar-faktischen Einfluss auf seine Willensbildung kommt. Dieser Einfluss ist nämlich allenfalls bei einem Teil der (politischen) Mitarbeiter des Abgeordneten überhaupt vorstellbar. Aber auch bei diesen hat er aufgrund der in der Regel überschaubaren Verzögerungen durch die Mitbestimmung kein solches Gewicht, das es rechtfertigen würde, hier von einem Eingriff in das Freie Mandat zu sprechen - dies umso weniger, als das BPersVG dem Abgeordneten mit der Möglichkeit vorläufiger Regelungen ein Instrument an die Hand gibt, sich vor einer von ihm nicht gewollten Einflussnahme auf die Freiheit seines Mandats selbst zu schützen.

\section{(c) Angemessene Amtsausstattung}

Es ist anerkannt, dass vom Schutz des Art. 38 Abs. 1 S. 2 GG solche separat normierten Rechte des Abgeordneten miterfasst werden, die seine Freiheit an erwiesenermaßen prekären Stellen absichern und die tatsächliche Grundlage für seine Unabhängigkeit schützen. Dazu gehört auch das Recht auf eine angemessene Entschädigung aus Art. 48 Abs. 3 S. 1

30 Wobei der Einfluss auf die Willensbildung des Abgeordneten nicht nur auf seiner wissenschaftlichen Arbeit, sondern wohl auch auf der „Filterfunktion“ der meisten wissenschaftlichen Mitarbeiter beruht. Vgl. dazu Volker Pilz, Moderne Leibeigenschaft? Berufsbild und soziale Absicherung der persönlichen Mitarbeiter der Bundestagsabgeordneten, in: ZParl, 35. Jg. (2004), H. 4, S. $667-681$, S. 669.

31 Vgl. ebenda, S. 675 f., der bei einer Kündigung nach Ablauf der Probezeit sogar eine Weiterbeschäftigung des Mitarbeiters bis zum Ende der Befristung für dem Abgeordneten zumutbar hält. 
$\mathrm{GG}^{32}$, zu der neben der Alimentation des Abgeordneten eine Erstattung von Ausgaben für eine angemessene Amtsausstattung zählt. ${ }^{33}$

Die Amtsausstattung beinhaltet gemäß $\$ 12$ Abs. 3 S. 1 AbgG auch die Aufwendungen für die Beschäftigung von Mitarbeitern zur Unterstützung der parlamentarischen Arbeit des Abgeordneten. Dass überhaupt Mitarbeiter zur Amtsausstattung eines Abgeordneten gehören, kann als zeitgemäße Konkretisierung des Art. 48 Abs. 3 GG gelten, nicht dagegen der konkrete Umfang; Einzelheiten dazu werden gemäß $₫ 12$ Abs. 1 S. 5 AbgG im Haushaltsgesetz und in vom Ältestenrat zu erlassenden Ausführungsbestimmungen ${ }^{34}$ geregelt. Kommt es durch die Mitbestimmung zu Verzögerungen bei Einstellungen oder Entlassungen oder der zeitlichen Einsetzbarkeit von Mitarbeitern und in Folge dessen zu einer faktischen Beeinträchtigung der parlamentarischen Arbeit des Abgeordneten, könnte man darin eine Verletzung des Anspruchs auf Amtsausstattung aus Art. 48 Abs. 3 S. 1 GG sehen. Dagegen spricht aber schon, dass der konkrete Umfang der Beschäftigung von Mitarbeitern nicht verfassungskräftig gewährleistet ist.

Des Weiteren garantiert Art. 48 Abs. 3 S. 1 GG dem Abgeordneten nur eine „angemessene" Amtsausstattung. Was angemessen ist, muss sich dabei am Schutzzweck der Norm orientieren, nämlich die Unabhängigkeit des Abgeordneten zu sichern. Eine Verletzung von Art. 48 Abs. 3 S. 1 GG durch das Mitbestimmungsrecht liegt daher allenfalls dann vor, wenn die Unabhängigkeit des Abgeordneten durch die Mitbestimmung rechtlich beeinträchtigt würde, und nicht schon dann, wenn ihm die Ausübung seiner parlamentarischen Arbeit bloß zeitweise erschwert wird. Dass durch mitbestimmungsrechtliche Verzögerungen die Amtsausstattung in Gestalt von Mitarbeitern - wenn auch nur zeitweise - auf ein Maß reduziert werden könnte, das die Unabhängigkeit des Abgeordneten gefährdet, erscheint aber praktisch ausgeschlossen, so dass auch insoweit eine Verletzung verfassungsrechtlicher Positionen des Abgeordneten nicht in Betracht kommt. Es handelt sich um bloße Belästigungen, die verfassungsrechtlich nicht relevant sind.

\section{Ergebnis}

Die Mitbestimmung nach dem BPersVG kollidiert nicht mit dem Freien Mandat gemäß Art. 38 Abs. 1 S. 2 GG. Mangels einer Kollision kommt es auch nicht zu einer auf dem Geltungsvorrang des Verfassungsrechts beruhenden verfassungsrechtlichen Sperrwirkung, das heißt das BPersVG ist auch im Verhältnis der Bundestagsabgeordneten zu ihren Mitarbeitern anwendbar.

32 Vgl. Norbert Achterberg / Martin Schulte, a.a.O. (Fn. 12), Art. 48 Rn. 44; Martin Morlok, a.a.O. (Fn. 16), Art. 38 Rn. 148.

33 Vgl. Bodo Pieroth, a.a.O. (Fn. 13), Art. 48 Rn. 7.

34 Die aktuell gültige Fassung ist seit dem 12. Oktober 2007 in Kraft. 